\title{
DESEMPENHO DE ESTUDANTES DE DIFERENTES MUNICÍPIOS EM UMA DISCIPLINA DO ENSINO SUPERIOR A DISTÂNCIA (CEDERJ)
}

\begin{abstract}
Glauca Torres Aragon | glauca.aragon@gmail.com
Bacharel em Geologia pela Universidade Federal do Rio de Janeiro, Doutora em Geociências pela Universidade Federal Fluminense, Professora da Universidade Estadual do Norte Fluminense Darcy Ribeiro (Centro de Biociências e Biotecnologia, Laboratório de Ciências Ambientais), Coordenadora de Disciplina "Dinâmica da Terra" do curso semipresencial de Licenciatura em Ciências Biológicas (CECIERJ/CEDERJ).
\end{abstract}

Cleide Ferreira da Silva | cleidefersilva@gmail.com

Bacharel em Biociências e Biotecnologia, Doutora em Biociências e Biotecnologia pela Universidade Estadual do Norte Fluminense, Coordenadora de Disciplina "Dinâmica da Terra" do curso semipresencial de Licenciatura em Ciências Biológicas (CECIERJ/CEDERJ).

\section{Resumo}

Este artigo descreve o desempenho de estudantes em uma disciplina do primeiro período da Licenciatura em Ciências Biológicas na modalidade semipresencial oferecida pela UFRJ, pela UERJ e pela UENF através da Fundação CECIERJ/Consórcio CEDERJ em 17 municípios do estado do Rio de Janeiro. O objetivo principal é comparar o desempenho de estudantes de diferentes polos regionais através de análise estatística das notas médias finais dos cursantes em cada polo, ao longo de seis semestres consecutivos. Os resultados indicam uma diferença significativa no desempenho de estudantes de diferentes polos regionais, indicando que o perfil diferenciado dos cursantes, especialmente relevante na modalidade de ensino a distância, pode incluir características regionais. Os diferentes estágios de desenvolvimento humano nos municípios-sede de cada polo regional são apresentados e correlacionados com indicadores de desempenho dos estudantes. Foi observada uma correlação positiva entre as notas finais médias dos cursantes em cada polo regional com as Taxas de Alfabetização e com o Índice de Desenvolvimento Humano Municipal - Dimensão Educação dos respectivos municípios-sede. Os resultados quantitativos são compatíveis com observações qualitativas realizadas durante as correções de provas dissertativas dos estudantes. Concluímos que a busca de mecanismos para reforço dos conteúdos do Ensino Fundamental e Médio é de especial relevância em municípios com baixos indicadores de desenvolvimento humano.

\section{Palavras-chave}

Ensino a distância. Desempenho de estudantes. Indicadores de Desenvolvimento Humano. 
Desempenho de estudantes de diferentes municípios em uma disciplina do ensino superior a distância (CEDERJ)

STUDENT PERFORMANCES IN A DISTANCE LEARNING HIGHER EDUCATION COURSE (CEDERJ) IN DIFFERENT MUNICIPALITIES

\section{Abstract}

This paper describes student performances in a first-semester subject of a distance learning Biological Sciences course, offered by UFRJ, UERJ and UENF through the CECIERJ Foundation/CEDERJ Consortium in 17 municipalities in Rio de Janeiro State. The main goal is to compare student performances at different regional centers through a statistical analysis of the final average grades of students at each center through six consecutive semesters. Results indicate a significant difference in student performances in different regional centers, indicating that the distinct student profiles, specially relevant in distance learning, might incorporate regional characteristics. Different human development index (HDI) stages of each regional center's municipality headquarters are presented and correlated to student performance indices. A positive correlation between final average grades in each regional center and the respective municipal headquarters' adult literacy rates and HDI-education index component was observed. Quantitative results are compatible with qualitative observations made during correction of student essay tests. We conclude that the search for performance improvement mechanisms among Elementary and High School students is particularly relevant to municipalities with low human development indices.

\section{Keywords}

Distance learning. Student performance. Human Development Index.

\section{Rendimiento de los estudiantes de distintas ciudades en una disciplina de la educación a distancia (CEDERJ).}

\section{Resumen}

Este estudio describe los resultados obtenidos por los estudiantes en una asignatura del primer período de la Licenciatura en Ciencias Biológicas ofrecido por la UFRJ, UERJ y UENF a través de la Fundación CECIERJ/CEDERJ en 17 municipios del Estado de Río de Janeiro. El objetivo principal es comparar el rendimiento de los estudiantes en los diferentes centros asociados de apoyo presencial a través del análisis estadístico de las puntuaciones medias finales de los estudiantes durante seis semestres consecutivos. Los resultados muestram una diferencia significativa en el desempeño de estudiantes en los distintos centros regionales asociados, que indican un perfil diferente de los estudiantes, que puede incluir las características regionales. Las distintas etapas del desarrollo humano en los distritos de la sede de cada centro asociado regional se presentan y se correlacionan con los indicadores de desempeño de los estudiantes. Se observó una correlación positiva entre el promedio de las calificaciones finales de los estudiantes en cada centro regional, con tasas de alfabetización y el Índice de Desarrollo Humano Municipal (nível educacional) de su sede local. Los resultados cuantitativos son consistentes con las observaciones cualitativas realizadas durante las correcciones de las preguntas de ensayo para los estudiantes. Llegamos a la conclusión de que la búsqueda de mecanismos para reforzar el contenido de la educación básica es de particular relevancia en los municipios con bajos indicadores de desarrollo humano.

\section{Palabras clave}

Educación a distancia. Desempeño de estudiantes. Índice de Desarrollo Humano.

\section{Introdução}

A Fundação CECIERJ/Consórcio CEDERJ foi criada com a união da autarquia Centro de Ciências do Estado do Rio de Janeiro - CECIERJ e do Centro de Educação a Distância do Estado do Rio de Janeiro CEDERJ. Desde 2006 a Fundação CECIERJ/Consórcio CEDERJ faz parte do Sistema Universidade Aberta do Brasil (UAB), e a maioria dos polos regionais e dos cursos passou a ser CEDERJ/UAB.

A estrutura pedagógica do CEDERJ integra momentos online e presenciais, sendo a estrutura composta por cinco pilares essenciais, a saber: 
a) material didático de apoio impresso e digital;

b) atendimento por tutores, composto por tutoria a distância e presencial;

c) estágios supervisionados;

d) processo de avaliação presencial nos polos;

e) práticas em laboratórios de disciplinas específicas nas áreas de Informática, Biologia, Física e Química nos polos regionais (SPÍNDOLA ET Al. ,2008).

A Licenciatura em Ciências Biológicas é oferecida em 17 polos, sendo o projeto acadêmico coordenado pela UFRJ (Universidade Federal do Rio de Janeiro), pela UENF (Universidade Estadual do Norte Fluminense Darcy Ribeiro) e pela UERJ (Universidade Estado do Rio de Janeiro). A matriz curricular é comum aos alunos de todos os polos, sendo as disciplinas divididas entre as três universidades. Cada universidade irá diplomar os alunos de um grupo de polos, e os alunos de todos os polos cursam disciplinas oferecidas pelas três universidades.

A disciplina Dinâmica da Terra (UENF/CEDERJ) foi oferecida semestralmente no período estudado (2006 a 2008) aos alunos de primeiro período da Licenciatura em Ciências Biológicas dos polos de Angra dos Reis (ARE), Bom Jesus do Itabapoana (BJE), Campo Grande (CGR), Itaperuna (ITA), Itaocara (ITO), Macaé (MAC), Nova Friburgo (NFR), Paracambi (PAR), Petrópolis (PET), Piraí (PIR), São Fidelis (SFI), São Francisco do Itabapoana (SFR), Três Rios (TRI) e Volta Redonda (VRE). A partir do primeiro semestre de 2007 (2007-1), foram incluídos os polos de Duque de Caxias (DCA), Nova Iguaçu (NIG) e Resende (RES).

A disciplina Dinâmica da Terra reúne fundamentos de geologia, limnologia e paleontologia, aprofundando e muitas vezes corrigindo conteúdos do Ensino Fundamental e Médio, situando-se entre as disciplinas do ciclo básico.

Sendo o material didático e as avaliações idênticos para os alunos de 17 municípios do RJ, o desempenho dos mesmos, quando analisado comparativamente entre os polos reflete as profundas diferenças regionais existentes no estado e aponta para a necessidade de promover a qualidade na formação de professores em diversos deles.

\section{Objetivos}

Analisar comparativamente o desempenho de estudantes em uma disciplina de primeiro período de um curso superior à distância (Licenciatura em Ciências Biológicas, CEDERJ) em 17 polos regionais do Estado do Rio de Janeiro. Buscar tendências regionais do perfil dos estudantes através da correlação entre o desempenho de estudantes de diferentes polos regionais e os respectivos indicadores de desenvolvimento humano.

\section{Material e métodos}

\subsection{Critérios de aprovação na disciplina}

A avaliação do desempenho dos estudantes segue as normas acadêmicas do CEDERJ (CEDERJ, 2009). As avaliações presenciais (APs) correspondem a $80 \%$ da nota final dos estudantes. As avaliações são idênticas para todos os polos, e realizadas simultaneamente. $\mathrm{O}$ mesmo vale para as avaliações a distância (ADs), que correspondem a $20 \%$ da média final. O material didático disponível é o mesmo para todos os estudantes, e são realizadas duas aulas práticas presenciais a cada semestre.

Todas as avaliações da disciplina analisada neste estudo foram compostas por questões discursivas, exigindo dos estudantes a capacidade de redigir respostas curtas com clareza.

\subsection{Indicadores de desempenho na disciplina}

Considerando os altos índices de evasão característicos do EAD, tomamos como indicadores do desempenho os seguintes índices:

- porcentagem de aprovados dentre os cursantes (excluindo os desistentes, que não fizeram nenhuma avaliação, e os que abandonaram a disciplina após a primeira avaliação presencial) em cada polo, por semestre;

- nota final média dos cursantes para todos os semestres de cada polo.

\subsection{Indicadores socioeconômicos dos municípios onde se localizam os diversos polos}

As bases de dados utilizadas neste estudo para obtenção dos indicadores de desenvolvimento humano dos municípios onde os polos estão localizados fo- 
ram o Atlas do Desenvolvimento Humano no Brasil (PNUD, 2000), o Portal IBGE Cidades (IBGE, 2006), Atlas de Dados Georreferenciados do Estado do Rio de Janeiro (CECIERJ, 2009) e o Armazém de Dados do Portal da Cidade do Rio de Janeiro (Prefeitura do Rio de Janeiro, 2009).

Essas bases de dados foram acessadas diversas vezes entre agosto de 2008 e julho de 2009, sendo a data do último acesso indicada nas referências. Os valores apresentados são referentes aos anos indicados em cada tabela.

Um dos indicadores escolhidos foi o PIB per capita (Produto Interno Bruto per capita), calculado pelo IBGE. O PIB per capita é definido como a razão entre o somatório da renda per capita de todos os indivíduos e o número total desses indivíduos. O PIB per capita é um indicador eficaz para a avaliação da renda de um universo amplo, como países e unidades da Federação. Sua eficácia é limitada quando aplicado a municípios, visto que muitos dos trabalhadores de um município podem residir em municípios vizinhos, distorcendo os resultados.

Outro índice escolhido foi o IDHM-E (Índice de Desenvolvimento Humano Municipal - dimensão educação), um dos subíndices componentes do IDH-M, calculado pelo PNUD. O IDH-M é uma adaptação do IDH (Índice de Desenvolvimento Humano) para populações menores, características de municípios.

O IDH mede o nível de desenvolvimento humano dos países a partir de indicadores de educação (alfabetização e taxa de matrícula), longevidade (esperança de vida ao nascer) e renda (PIB per capita). Para aferir o nível de desenvolvimento humano de municípios as dimensões são as mesmas - educação, longevidade e renda -, mas alguns dos indicadores usados são diferentes, embora meçam os mesmos fenômenos.

Para medir o acesso à educação da população de uma localidade, o IDHM-E considera dois indicadores: a porcentagem de pessoas alfabetizadas entre os moradores com mais de 15 anos de idade daquele lugar (com peso dois no cálculo final) e a taxa de frequência bruta a salas de aula (peso um).

A Taxa de Alfabetização de maiores de 15 anos (que compõe o IDHM-E) foi também considerada separa- damente, por sua importância na identificação de características educacionais regionais.

\subsection{Estatística}

A análise estatística dos dados foi feita com auxílio do programa StatPlus e consistiu em análise de variância das notas finais obtidas por todos os cursantes de cada polo no período de 2006 a 2008 e na correlação pelo coeficiente de Pearson $(r)$ entre as notas finais médias dos cursantes de cada polo e com o IDHM-E e com as taxas de alfabetização dos respectivos municípios-sede.

\section{Resultados e discussão}

O crescente acesso aos cursos de nível superior no Brasil é responsável por avanços sociais importantes, particularmente no caso da EAD. A adaptação à vida universitária, no entanto, apresenta muitas dificuldades para estudantes com dificuldades de leitura e produção de textos. Segundo Sampaio e Santos (2002) as deficiências de compreensão e o escasso hábito de leitura entre universitários são responsáveis, em grande parte, pelo baixo desempenho do alunos.

As desigualdades em nossa sociedade são muitas, e para alcançar o objetivo de oferecer uma educação universitária inclusiva é necessário contemplar não só a exclusão digital, mas também deficiências na formação básica.

\subsection{Os indicadores de desenvolvimento no Brasil e nos municípios do estado do Rio de Janeiro}

O IDH do Brasil vem evoluindo positivamente nos últimos 30 anos. Em 2005, atingiu o valor de corte de 0,800 e passou a ser incluído entre os países com desenvolvimento humano elevado, de acordo com o Relatório do Desenvolvimento Humano 2007/2008 publicado pelo PNUD (2009).

Essa tendência de melhora foi acompanhada pelos municípios do estado do Rio de Janeiro (PNUD, 2000), sendo a média dos municípios do RJ superior à dos municípios do Brasil. Entretanto, as desigualdades entre municípios (tanto do Brasil como do RJ) são ainda muito profundas. 
As bases de dados disponíveis para os municípios do Estado do Rio de Janeiro onde estão instalados os polos regionais do CEDERJ são referentes ao período de 1991 a 2000 (PNUD, 2000), e sendo assim optamos por considerar os valores relativos ao ano 2000, anteriores à implantação do CEDERJ. Dessa forma, nossa análise não pretende avaliar o possível impacto da oferta de licenciaturas na modalidade EAD através do Consórcio CEDERJ nesses municípios. Neste estudo o desempenho dos discentes em uma disciplina é discutido considerando os indicadores de desenvolvimento anteriores à implantação do CEDERJ.

A Tabela 1 apresenta os indicadores de desenvolvimento selecionados para os municípios onde é oferecida a Licenciatura em Ciências Biológicas através do Sistema CEDERJ. A base de dados relativos ao PIB per capita corresponde ao ano 2006 (IBGE, 2006). Para o IDHM-E as bases de dados disponíveis são referentes ao ano 2000 (PNUD, 2000). Os municípios estudados estão agrupados de acordo com as regiões governamentais disponíveis no Atlas de Dados Georreferenciados do Estado do Rio de Janeiro (CECIERJ, 2009).

O PIB per capita varia de $\mathrm{R} \$ 6.673,00$ em São Francisco do Itabapoana a $\mathrm{R} \$$ 40.281,00 em Macaé (Tabela 1). O município de Macaé se destaca dos demais, com o PIB per capita fortemente influenciado pela atividade de prospecção de petróleo da bacia de Campos.

Da mesma forma que o PIB per capita, o IDHM-E também apresentou uma grande faixa de variação, refletindo as desigualdades entre os municípios do RJ, com valor mínimo na Região Norte Fluminense (0,715 em São Francisco do Itabapoana) e máximo na Região do Médio Paraíba $(0,931$ em Volta Redonda).

Tabela 1: Indicadores de desenvolvimento dos municípios-sede dos polos regionais do CEDERJ incluídos neste estudo

\begin{tabular}{|c|c|c|c|c|}
\hline $\begin{array}{l}\text { Região } \\
\text { Governamental }\end{array}$ & Município/polo CEDERJ & $\begin{array}{l}\text { PIB per capita, } \\
\text { valores em reais, } \\
\qquad 2006\end{array}$ & $\begin{array}{l}\text { IDHM-E, } \\
2000\end{array}$ & $\begin{array}{c}\text { Taxa de } \\
\text { alfabetização (\%) }\end{array}$ \\
\hline \multirow{4}{*}{ Metropolitana } & Rio de Janeiro/CGR & 7.020 & 0,920 & 95,8 \\
\hline & Duque de Caxias/DCA & 26.392 & 0,873 & 92,4 \\
\hline & Nova Iguaçú/NIG & 7.418 & 0,884 & 93,2 \\
\hline & Paracambi/PAR & 6.839 & 0,897 & 90,3 \\
\hline \multirow{3}{*}{$\begin{array}{l}\text { Noroeste } \\
\text { Fluminense }\end{array}$} & $\begin{array}{c}\text { Bom Jesus do Itabapoana/ } \\
\text { BJE }\end{array}$ & 7.855 & 0,851 & 87,5 \\
\hline & Itaocara/ITO & 9.686 & 0,837 & 87,8 \\
\hline & Itaperuna/ITA & 13.207 & 0,859 & 88,8 \\
\hline \multirow{3}{*}{ Norte Fluminense } & Macaé/MAC & 40.281 & 0,889 & 92,7 \\
\hline & São Fidélis/SFI & 7.020 & 0,822 & 85,3 \\
\hline & São Francisco/SFR & 6.673 & 0,715 & 77,4 \\
\hline \multirow{2}{*}{ Região Serrana } & Nova Friburgo/NFR & 10.853 & 0,885 & 93,1 \\
\hline & Petrópolis/PET & 14.584 & 0,888 & 94 \\
\hline \multirow{3}{*}{ Médio Paraíba } & Piraí/PIR & 30.960 & 0,875 & 90 \\
\hline & Resende/RES & 26.137 & 0,918 & 94 \\
\hline & Volta Redonda/VRE & 23.269 & 0,931 & 95 \\
\hline $\begin{array}{l}\text { Centro Sul } \\
\text { Fluminense }\end{array}$ & Três Rios/TRI & 11.660 & 0,893 & 93 \\
\hline Baía de Ilha Grande & Angra dos Reis/ARE & 24.250 & 0,870 & 92 \\
\hline
\end{tabular}

Fontes: Para o PIB per capita, IBGE, 2006; para o IDHM-E, PNUD, 2000; e para a taxa de alfabetização, CECIERJ, 2009. No caso do polo Campo Grande, o PIB per capita se refere ao município do Rio de Janeiro (IBGE, 2006) e o IDHM-E se refere à XVIII Região Administrativa da Cidade do Rio de Janeiro, (Prefeitura do Rio de Janeiro, 2009). 


\subsection{Descrição do desempenho discente na} disciplina em diferentes polos CEDERJ onde a mesma foi oferecida entre 2006 e 2009

Em nossa análise não consideramos o porcentual de discentes residentes em municípios vizinhos a diversos polos, o que deverá ser quantificado posteriormente para um aprofundamento deste estudo. Através do depoimento de diversos tutores presenciais, sabe-se que esse porcentual não é desprezível em diversos polos.

São exemplos os polos BJE (onde existem diversos estudantes oriundos de cidades do estado do Espírito Santo), SFR e SFI (nos quais muitos dos alunos residem na cidade de Campos dos Goytacazes) e ARE (que conta com diversos estudantes de cidades do estado de São Paulo).

O total de alunos inscritos na disciplina Dinâmica da Terra aumentou a partir do primeiro semestre de 2007 (2007-1) com o início do funcionamento dos polos DCA, NIG e RES (Figura 1).

Esse número bruto, entretanto, não reflete o número de estudantes que realmente cursaram a disciplina, visto que muitos não chegam a fazer nenhuma avaliação, sendo considerados desistentes.
Um outro caso corresponde aos estudantes que decidem abandonar a disciplina em um momento posterior, após a primeira avaliação presencial. Esses alunos incluem os que chegam a experimentar o curso superior na modalidade EAD e optam por outros cursos na modalidade presencial e os que desistem apenas de fazer as avaliações da disciplina, permanecendo no curso e retornando à disciplina em um momento posterior.

Dentre os fatores que contribuem para este quadro, destacamos a dificuldade de adaptação à nova metodologia de ensino, a dificuldade de locomoção até os polos, a falta de conhecimentos mínimos de informática e o desconhecimento, por parte do estudante, da necessidade de dedicar um grande número de horas semanais ao estudo.

Neste estudo foi considerada "desistência" o caso do discente que não fez nenhuma avaliação da disciplina e "abandono" o caso do discente que não realizou avaliações após a primeira AP.

O número de inscritos, excluindo-se os casos de desistência e abandono, corresponde ao número de discentes cursantes na disciplina (Figura 1).

Figura 1: Total de estudantes inscritos e cursantes na disciplina no período de 2006 a 2008 (somatório para todos os polos)

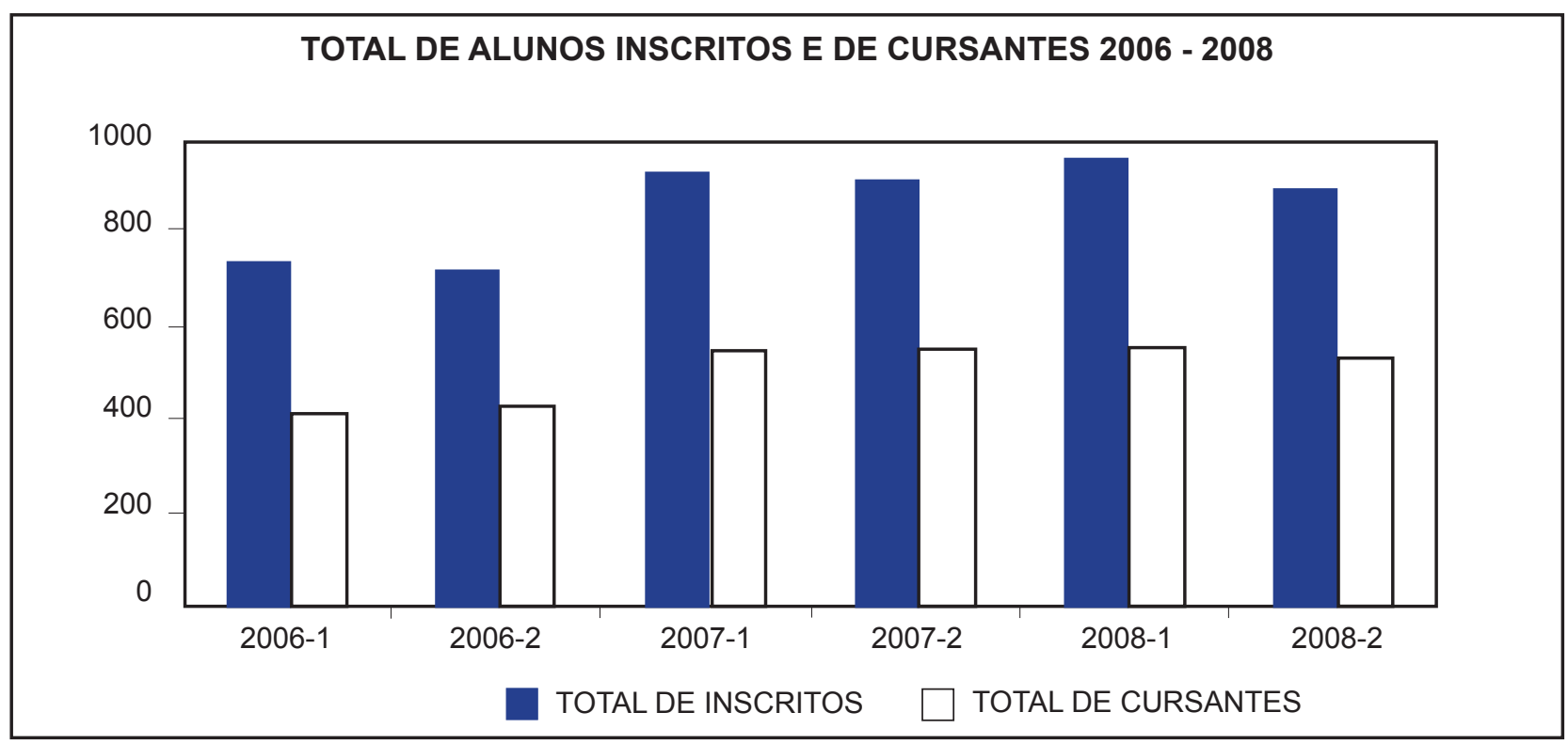

As taxas de desistência e abandono somadas são muito altas, com ampla faixa de variação entre os polos e para cada polo em diferentes semestres (Figura 2). As motivações em ambos os casos são variadas, não sendo possível analisá-las sem aprofundar este estudo, incluindo uma caracterização socioeconômica dos estudantes incluídos em cada caso. As variadas porcentagens de abandono e desistência afetam de modo significativo as médias finais, mascarando o desempenho obtido pelos estudantes considerados cur- 
santes, objeto deste estudo. Por esse motivo, na análise das notas finais consideramos apenas os cursantes.

As médias finais dos discentes cursantes por polo no período estudado (2006-2008) são apresentadas na Figura 3, que apresenta ainda a nota final média para todos os cursantes de cada polo no período estudado.
A análise de variância (ANOVA) indicou uma diferença significativa entre as notas finais obtidas por estudantes dos diferentes polos em estudo $(\mathrm{F}=15,156 \mathrm{p}<0,01)$, considerando-se o conjunto de todas as notas finais dos cursantes de cada polo.

Figura 2: Porcentagem de desistentes por polo no período estudado, com faixas de variação representando valores máximos e mínimos

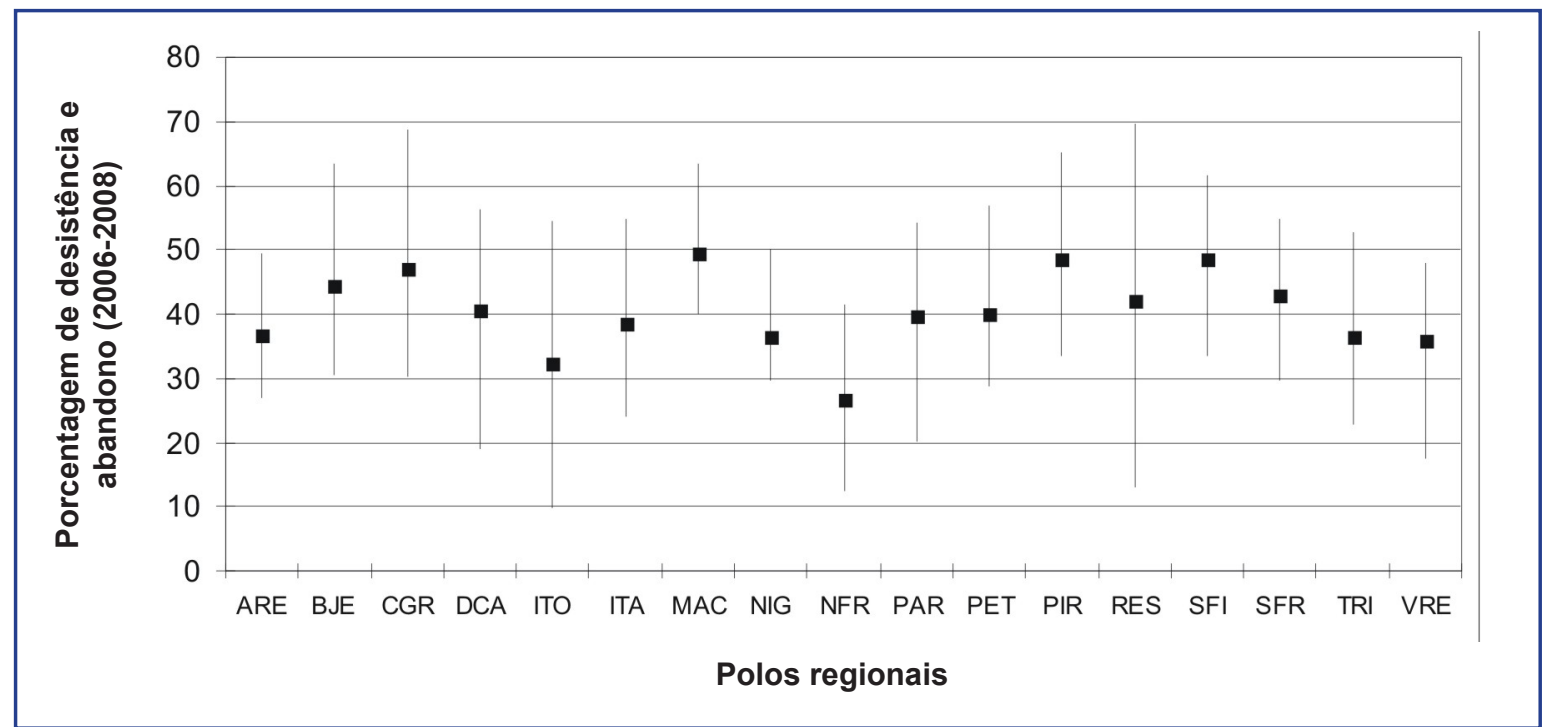

Figura 3: Médias das notas finais dos discentes de cada polo, por semestre, e média geral para todos os cursantes em cada polo no período estudado (com faixa de variação de mais e menos 1 desvio padrão)

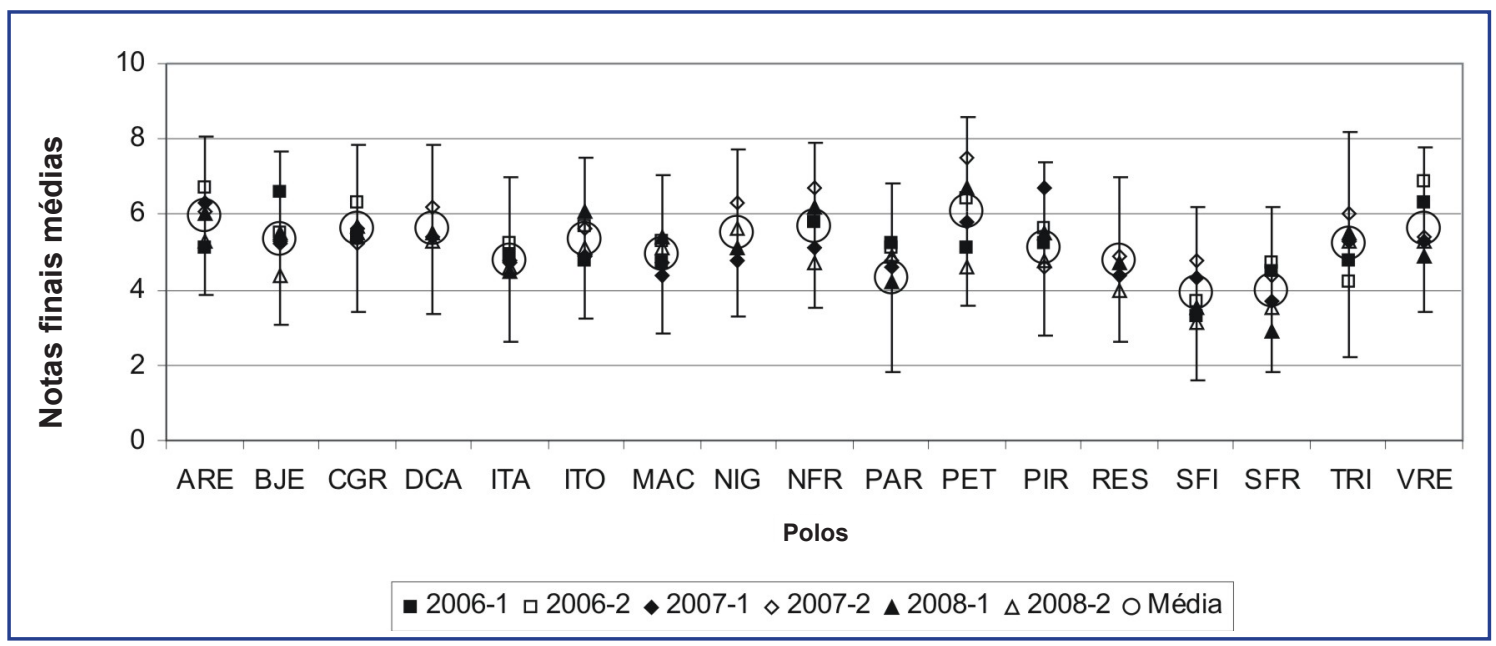

\subsection{Correlações entre índices de}

\section{desenvolvimento humano dos municípios e} indicadores de desempenho dos estudantes

A Figura 4 ( $a$ e b) apresenta a dispersão obtida para as relações entre as notas finais médias dos cursantes de cada polo e os respectivos IDHM-E e taxas de alfabetização dos municípios sede. Os valo- res do coeficiente $r$ (Pearson) obtidos (0,5087 para o IDHM-E e 0,6847 para as taxas de alfabetização) foram significativos ao nível p 0,05 ( $r$ crítico 0,4555 para $\mathrm{n}=17$ ).

A correlação observada entre as taxas de alfabetização dos municípios-sede dos polos e as médias das notas finais dos estudantes dos respectivos po- 
los reflete a influência da capacidade leitora dos estudantes sobre seu aprendizado, expondo as deficiências na formação básica dos estudantes nos municípios mais carentes. Embora todos os estudantes tenham concluído o ensino médio, observa- se, durante a correção das avaliações, uma grande dificuldade na leitura, interpretação de textos e redação por parte dos estudantes, em especial nos polos de municípios com baixas taxas de alfabetização e baixos índices IDHM-E.

Figura 4: Dispersão obtida entre as taxas de alfabetização (a) e o IDHM-E (b) dos municípios sede dos polos e as notas finais médias para todos os cursantes dos respectivos polos

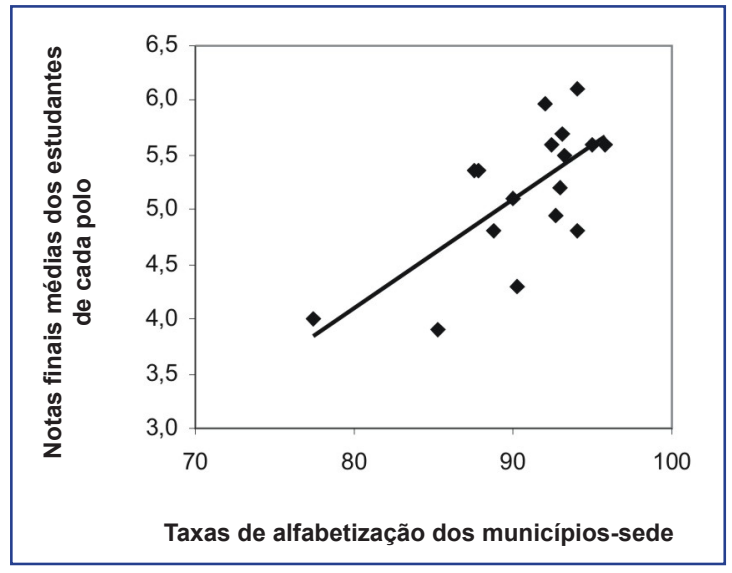

As estatísticas disponíveis relacionadas à educação refletem dados quantitativos tais como a taxa de alfabetização e taxas brutas de frequência à escola. Esses números ajudam a identificar municípios mais carentes de investimentos na área de educação. Entretanto, quando analisamos o desempenho de estudantes universitários em uma mesma disciplina em diferentes municípios, o resultado reflete a qualidade da educação básica (ensino fundamental e médio) nos municípios considerados.

Inúmeros trabalhos discutem a capacidade das escolas de formar leitores-alunos maduros, críticos, competentes e reflexivos como preconizam os novos Parâmetros Curriculares da Língua Portuguesa MEC/ Brasil (1997). Ferreira e Dias (2002) observaram que a escolarização prolongada não tem se mostrado um fator suficiente para garantir o bom desempenho do indivíduo em atividades de compreensão de leitura.

Nossos resultados sugerem que essa questão é ainda mais crítica em municípios com menores taxas de alfabetização e baixos IDHM-E.

As dificuldades relativas ao aprendizado e à formação de leitores críticos e reflexivos refletem ainda a concepção comportamentalista da educação, tão presente ainda hoje nas salas de aula. Ao criticar o que

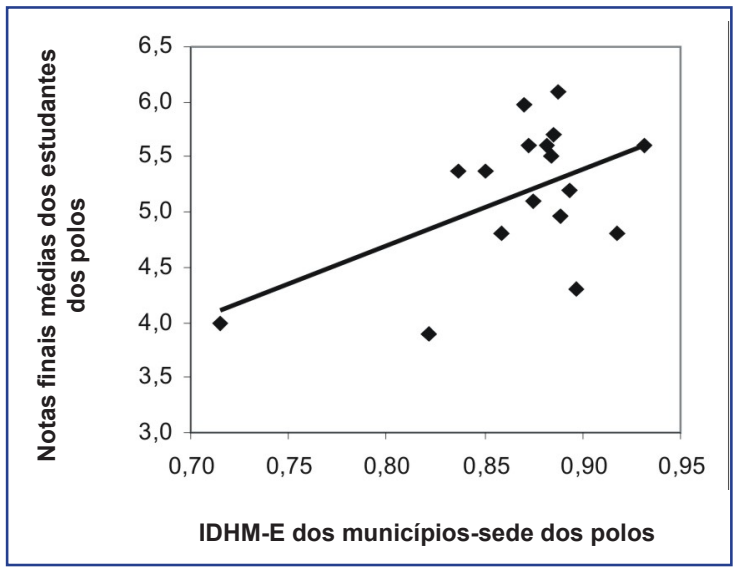

chamou de "concepção bancária" da educação, Freire (2005) afirma:

Desta maneira, a educação se torna um ato de depositar, em que os educandos são os depositários e o educador o depositante. Em lugar de comunicar-se, o educador faz 'comunicados' e depósitos que os educandos, meras incidências, recebem pacientemente, memorizam e repetem.

De acordo com Campos et al. (2007), existem muitos modelos de EAD, combinando diferentes recursos e formas de comunicação. Segundo esses autores:

Nesse contexto, a distância pode ser vista como um elemento positivo para o desenvolvimento da autonomia da aprendizagem, permitindo que o estudante assimile o conhecimento em seu próprio ritmo. Já os portais temáticos reúnem em um mesmo espaço virtual informações selecionadas sobre um determinado assunto ou campo do conhecimento, oferecendo formas de comunicação entre seus usuários...

O ensino superior a distância representa uma mudança de paradigma em relação ao ensino, que deixa de ser centrado no professor para ser centrado no aluno. O material didático escrito propicia ao aluno vivenciar a aprendizagem através da leitura, tornandose mais independente. Barreto (2007) destaca:

$O$ estudo de um texto é um processo cujo ritmo é inteiramente ditado pelo aluno. Se, à primeira leitura, algum conceito lhe escapou à compreensão, ele pode retomar a passagem quantas vezes quiser. 
Dessa forma, fica clara a importância de oferecer um ensino com concepção problematizadora em $\mathrm{EAD}$, capacitando os docentes envolvidos a aproveitar os recursos disponíveis para desenhar cenários construtivistas e sociointeracionistas. $\mathrm{O}$ material didático impresso, neste contexto, constitui uma ferramenta de inclusão social que deve ser aproveitada para a formação de futuros professores-leitores críticos, maduros e reflexivos.

\section{Conclusões}

A formação de professores através do ensino superior a distância favorece a fixação dos profissionais formados em seus municípios de origem. Além disso, é uma proposta capaz de levar os futuros professores a vivenciar a aprendizagem através da leitura, contribuindo para melhorar a capacidade leitora dos mesmos e possuindo, dessa forma, um grande potencial de melhoria na qualidade do ensino básico em médio prazo.

Informalmente, foram observadas diferenças no desempenho médio dos alunos cursantes da disciplina em diferentes polos. A análise de variância confirmou a diferença entre os resultados obtidos por estudantes cursantes de diferentes polos. As notas finais médias para cursantes de cada polo apresentaram uma cor- relação positiva significativa com o IDHM-E e com as taxas de alfabetização dos municípios-sede.

As dificuldades observadas na leitura, interpretação e redação de textos, mais frequentes entre cursantes de municípios com baixas taxas de alfabetização, deverão ser mais bem investigadas, confrontando-se os resultados obtidos nesta disciplina com indicadores de desempenho em outras disciplinas e cursos.

Uma importante alteração no critério de inscrição na disciplina em estudo foi introduzida no primeiro semestre de 2009. Os alunos com nota geral inferior a 5 no vestibular passaram a ser inscritos em duas novas disciplinas: Fundamentos de Matemática, Física e Química e Seminários em EAD, cursando Dinâmica da Terra apenas no segundo semestre. As disciplinas introduzidas têm por objetivo reforçar os conteúdos do Ensino Médio e desenvolver a capacidade leitora e de redação dos estudantes. Os resultados dessa modificação deverão ser avaliados posteriormente, visto que ainda não existem dados suficientes para análise.

As causas das altas taxas de desistência e abandono também precisam ser mais bem compreendidas, procurando-se identificar medidas mitigadoras que favoreçam a permanência dos estudantes no Ensino Superior sem prejuízo da qualidade do ensino. 


\section{Referências bibliográficas}

BARRETO, Cristine Costa et al. Material impresso como recurso educacional: isso é história? In: Planejamento e Elaboração de Material Didático Impresso para Educação a Distância. Rio de Janeiro: BRASIL/ MEC, /UAB, 2007.

BRASIL. MEC/Parâmetros Curriculares da Língua Portuguesa. 1997. Disponível em: < http://portal.mec. gov.br/seb/arquivos/pdf/livro02.pdf> . Acesso em: 08 maio 2009.

FERREIRA, S. P. A.; DIAS, M. G. B. B. A escola e o ensino da leitura. Psicologia em estudo, Maringá, v. 7, n. 1, p. 39-49, 2002.

FUNDAÇÃO CECIERJ. Atlas de Dados Georreferenciados do Estado do Rio de Janeiro. 2009. Disponível em: <http:/www.cederj.edu.br/atlas/>. Acesso em: 30 maio 2009.

FUNDAÇÃO CEDERJ. Plataforma virtual CEDERJ/ CECIERJ. 2009. Disponivel em: <http://www.cederj. edu.br/fundacaocecierj/>. Acesso em: 30 jul. 2009.

IBGE.IBGE Cidades@.2006. Disponível em: < http:// www.ibge.gov.br/cidadesat/topwindow.htm?1>. Acesso em: 04 jun. 2009.

PNUD. Atlas do Desenvolvimento Humano no Brasil. 2000. Disponível em: <http://www.pnud.org.br/ atlas/>. Acesso em: 12 maio 2009.

PNUD. Relatório do Desenvolvimento Humano 2007/2008. 2009. Disponível em: < http://www.pnud. org.br/rdh/> . Acesso em: 01 jul. 2009.

RIO DE JANEIRO. Prefeitura. Portal do Município do Rio de Janeiro, Armazém de Dados, Bairros Cariocas. 2009. Disponível em: < http://portalgeo.rio. rj.gov.br/bairroscariocas/index_ra.htm $>$. Acesso em: 03/07/2009.
SAMPAIO, I.S.; SANTOS, A. A. A. Leitura e redação entre universitários: avaliação de um programa de intervenção. Psicologia em estudo, Maringá, v. 7, n. 1, p. 31-38, 2002.

SPÍNDOLA, Márcia; MOUSINHO, Sílvia Helena; SILVA, Rosemaria Vieira. EAD: o CEDERJ e a construção de uma prática pedagógica inclusiva. In: CREAD MERCOSUR/SUL, 12., 2008, Rio de Janeiro. Anais... Disponível em: <http:/www.cread2008.com. br/programacao/trabalhos/1041.pdf> . Acesso em: 10 jul. 2009. 